\title{
Calibration and standardisation of synchrotron radiation and conventional circular dichroism spectrometers. Part 2: Factors affecting magnitude and wavelength
}

\author{
Andrew J. Miles a ${ }^{\text {, Frank Wien }}{ }^{\text {a }}$, Jonathan G. Lees ${ }^{\mathrm{a}, *}$ and B.A. Wallace ${ }^{\mathrm{a}, \mathrm{b}, * *}$ \\ ${ }^{a}$ Department of Crystallography, Birkbeck College, University of London, London WC1E 7HX, \\ United Kingdom \\ ${ }^{\mathrm{b}}$ Centre for Protein and Membrane Structure and Dynamics, Daresbury Laboratory, \\ Warrington WA4 4AD, United Kingdom
}

\begin{abstract}
Circular dichroism (CD) spectroscopy is an important tool in structural biology, especially for protein secondary structure analyses. Synchrotron radiation circular dichroism (SRCD) spectroscopy is a modified version of the technique that uses the intense light from a synchrotron source to enable the collection of data to much lower wavelengths than possible on conventional circular dichroism (cCD) instruments. There is a need for standardization of calibration methods amongst and between CCD and SRCD instruments to ensure consistency and the ability to use common reference databases for empirical secondary structural analyses. In a previous study (Spectroscopy 17 (2003), 653-661), we compared optical rotation measurements on several CCD and SRCD instruments, whilst holding constant other experimental factors. In this study, other experimental parameters which contribute to the spectral magnitude, such as cell pathlength and protein concentration determinations, are examined. In addition, the extent of wavelength calibration variations between instruments and their effects on secondary structure calculations have been examined. Hence, this paper provides additional practical guidance for "good practice" in the measurement of CD data.
\end{abstract}

\section{Introduction}

Circular dichroism (CD) spectroscopy is a long-established technique for examining the secondary structure of proteins and for monitoring protein interactions and folding pathways. It is gaining acceptance as a technique approved for the characterization of macromolecules for pharmacological use. Synchrotron radiation-based circular dichroism (SRCD) spectroscopy made its debut more than 20 years ago [1,2], but its full potential for the study of protein structures has only recently been exploited. The intense light from the synchrotron source permits the collection of higher quality data than that obtained using conventional circular dichroism (cCD) instruments and appreciably extends the lower wavelength limit of the data [3]. It has been suggested that the extra information available in the vacuum ultraviolet (VUV) data will allow more accurate discernment of structural features, potentially even including information on folds and motifs [4]. To this end, new reference databases of circular dichroism spectra which include the lower wavelength data and a wider base of protein structural types, are being created

\footnotetext{
*Present address: School of Biological Sciences, Queen Mary, University of London, London E1 4NS, United Kingdom.

** Corresponding author. Tel.: +44 (0)207 631 6800; Fax: +44 (0)207 631 6803; E-mail: ubcg25a@ mail.cryst.bbk.ac.uk.
} 
[4,5] to enable improved analyses. For these to be useful with both CCD and SRCD data, it is necessary to establish a consistent set of calibration standards and protocols to ensure compatibility between and amongst SRCD and cCD instruments.

In a previous study [6], the optical rotation magnitude measurements for a number of standard compounds plus one protein were measured on several SRCD and CCD instruments, whilst holding other potential variables (sample, sample cell, measurement parameters, instrument operator, etc.) constant in order to strictly examine the extent of variation between instruments. Based on this, a method was proposed for the standardisation of protein spectra, to "correct" for the instrumental variations $[6,7]$.

However, in addition to instrumental variations, errors in spectral magnitude arising from other sources can produce serious errors in protein secondary structures calculated from CD data [8]. Potential sources of magnitude error that were not tested in the previous study because the same sample in the same cell was used for all measurements, are the determinations of protein concentration and cell pathlength. In the present study two popular methods for measuring protein concentration, the $A_{280}$ in the presence of denaturant [9] and quantitative amino acid analysis (QAA) are compared. In addition, two methods for measuring cell pathlengths, the interference fringe method [10] and the chromate dilution method are compared.

Another source of instrumental error can arise from misalignment or miscalibration of the instrument monochromator, which can lead to apparent shifts of wavelength that are manifest as differences in peak positions. These, too, will have a significant effect on empirical analyses of protein spectra. In this study we not only show the effects of wavelength shifts on the analyses of secondary structure, but also examine simple means of accurately calibrating instruments to eliminate this source of error. If an instrument spectral resolution of at least $0.2 \mathrm{~nm}$ could be achieved, benzene vapour was used as it produces a series of sharp peaks between $270 \mathrm{~nm}$ and $230 \mathrm{~nm}$ [11]. In addition, a certified holmium oxide filter was used to measure the wavelength variations in the near UV and visible regions; the results obtained by these two methods were compared.

In summary, this paper reports on a number of practical issues that can affect measurements of cCD and SRCD spectra, and describes means of accurately determining parameters which contribute to spectral magnitude, such as protein concentration and cell pathlength.

\section{Materials and methods}

\subsection{Materials}

Horse skeletal myoglobin was purchased from ICN Biochemicals, chicken lysozyme from Worthington, human serum ceruloplasmin from Calbiochem (USA). Human serum albumin (HSA), jackbean concanavalin A (Con A), and chicken avidin were from Sigma-Aldrich Company Ltd. All proteins had a purity of $>95 \%$. Prior to use, each sample was dialysed, centrifuged and degassed as described in Miles et al. [6].

Benzene (AnalaR grade) was obtained from BDH Laboratory supplies, UK. (+)-Camphor-10-sulfonic acid, $99 \%$ purity, guanidine hydrochloride, $>99 \%$ purity, and potassium chromate $\left(\mathrm{K}_{2} \mathrm{CrO}_{4}\right), 99.99 \%$ purity, were obtained from Sigma-Aldrich Company Ltd. A certified holmium oxide glass filter from Hellma UK Ltd. was used.

\section{2. $C D$ and SRCD spectroscopic measurements}

cCD measurements were made on an Aviv 62Ds, an Aviv 215, a JASCO 600 and a JASCO 715 instrument. SRCD measurements were made at the SRS Daresbury (UK) on beamline CD12; at ISA (Den- 
mark) on beamline UV1; at the NSLS (USA) on beamlines U9b and U11; and at BESSY2 (Germany) on beamline 3m_NIM1_C.

The holmium oxide glass filter was scanned from 600 to $200 \mathrm{~nm}$ on the cCD instruments but only from 400 to $200 \mathrm{~nm}$ on the SRCD instruments, due to limitations in their high wavelength ranges. Other parameters used were: bandwidth $1 \mathrm{~nm}$, step size $0.1 \mathrm{~nm}$, averaging/dwell time $0.2 \mathrm{sec}$ and temperature $25^{\circ} \mathrm{C}$. The benzene sample was prepared by introducing one drop of benzene into a $1 \mathrm{~cm}$ cuvette, which was then tightly stoppered. The high tension (HT) or dynode signal of the vapour was recorded over the wavelength range from 270 to $240 \mathrm{~nm}$ with a step size of $0.1 \mathrm{~nm}$, an averaging/dwell time of $0.2 \mathrm{sec}$ and at temperature of $25^{\circ} \mathrm{C}$. The bandwidth was set to $0.1 \mathrm{~nm}$ on three CCD instruments and on UV1, at $0.2 \mathrm{~nm}$ (the lowest possible). The narrowest possible bandwidth was used on 3m_NIM1_C, which had no calibrated bandwidth control at the time. CD12, U96 and U11 did not allow resolutions of lower than $0.5 \mathrm{~nm}$ therefore the sharp benzene peaks could not be measured on them accurately. The protein spectra and baselines were collected using the same (nominally) $0.001 \mathrm{~cm}$ pathlength circular demountable cell (Hellma UK Ltd.) over the wavelength range from 280 to $178 \mathrm{~nm}$ on conventional instruments and over the range from 280 to $168 \mathrm{~nm}$ on SRCD machines. On all instruments the bandwidth was set at $1 \mathrm{~nm}$, step size $0.2 \mathrm{~nm}$, averaging/dwell time $1 \mathrm{~s}$, and temperature $4^{\circ} \mathrm{C}$. An average of 3 scans was used in each case.

\subsection{Secondary structure calculations}

The secondary structural analyses used the DICHROWEB http://www.cryst.bbk.ac.uk/cdweb interactive webserver [12] with the CONTINLL [13] algorithm and database 1 [14].

\subsection{Sample cell pathlength determinations}

A UV/VIS spectrophotometer (CARY 3) was used in transmission mode, with a step size of $0.2 \mathrm{~nm}$, bandwidth of $1 \mathrm{~nm}$, and an air baseline collected between 650 and $350 \mathrm{~nm}$, followed by a scan of the empty cell. The pathlengths of a number of cells were determined according to the formula given in the legend for Fig. 1.

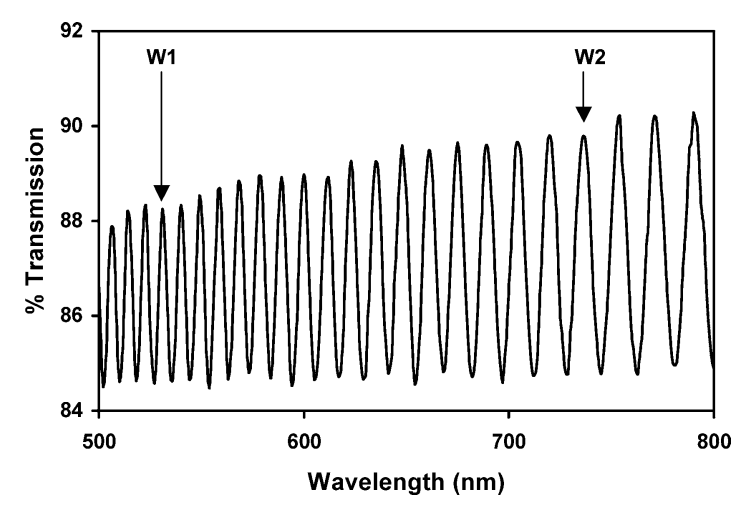

Fig. 1. Interference fringes obtained by scanning an empty sample cell with a nominally $0.001 \mathrm{~cm}$ pathlength using an UV/VIS spectrometer in transmission mode. The cell pathlength, calculated from the formula $\operatorname{PL}(\mu \mathrm{m})=$ $[(n(W 1 * W 2)) /(2(W 2-W 1))] / 1000$, where $W 1$ and $W 2$ are the wavelengths of the first and last fringes in $\mathrm{nm}$ and $n$ is the number of fringes between the $W 1$ and $W 2$. 
For the chromate dilution method, 1 litre of $0.02 \mathrm{M}$ potassium chromate in $0.01 \mathrm{M} \mathrm{NaOH}$ was prepared gravimetrically. $0.002 \mathrm{M}$ and $0.0002 \mathrm{M}$ solutions were prepared by serial dilution. Pathlengths were determined using $\varepsilon_{372}=4830 \mathrm{moll}^{-1} \mathrm{~cm}^{-1}[15]$.

\subsection{Protein concentration determinations}

For each sample, the protein concentration was measured using the absorbance method of Gill and von Hipple [9] in the presence of guanidine hydrochloride. In addition, two separate $10 \mu \mathrm{l}$ aliquots of each protein sample were examined by quantitative amino acid analysis (QAA) at the PNAC facility, University of Cambridge.

\section{Results}

\subsection{Sample cell pathlength determinations}

To achieve the full potential of SRCD instruments to measure low wavelength data, it is necessary to use short demountable pathlength cells $(\leqslant 0.005 \mathrm{~cm})$ to minimize the absorption due to water, buffer, and salts. Whilst the pathlengths of long cells $(0.1-1.0 \mathrm{~cm})$ are usually accurately reported by the manufacturers, the reported pathlengths of short cells can be in error by as much as $50 \%$, which in turn will result in this level of error in the spectral magnitudes. Accurate measurement of the cell pathlength can be achieved by using the interference fringe method [10]. This relies on a portion of the incident beam being retarded by double reflection from the inner surfaces of the empty cell. An interference pattern is produced in the visible and UV transmission spectrum (Fig. 1) when the retarded beam interferes with the main beam and the distance between the peaks is inversely proportional to twice the cell pathlength. When measuring the cell pathlength, the cell must be loaded into the same cell holder used when measuring the CD spectrum with the two halves of the cell in the same orientation relative to each other to achieve reproducible results. It should be noted that this method gives a minimum pathlength for a demountable cell since a thin layer of liquid can form between the flanges when a sample is added, increasing the pathlength by $0.5-1 \mu \mathrm{m}$, although this can be avoided by careful loading with the smallest possible sample volume so it does not overflow into the spacer region, or by designing cells with a different loading geometry (Wien and Wallace, in preparation). In this study the pathlengths of a number of nominally $10 \mu \mathrm{m}$ quartz Suprasil cells were found to range from 12 to $16 \mu \mathrm{m}$, while cells reported as being $4 \pm 1 \mu \mathrm{m}$ were found to be between 6 and $7 \mu \mathrm{m}$. In the latter case this is a $75 \%$ error. Very similar results were obtained using the potassium chromate dilution method, with the results deviating from the fringe results by only $2-3 \%$. However, for very short pathlength cells, the interference fringe method is more accurate as it is not dependent on precise measurements of small absorbances.

\subsection{Protein concentration determinations}

The concentrations of a number of proteins were measured both by using their absorbance at $280 \mathrm{~nm}$ (in $6 \mathrm{M}$ guanidine hydrochloride) and by quantitative amino acid analysis (Table 1). For the QAA measurements, two aliquots of each protein sample were examined, and in the worse cases there was a difference of $\sim 5 \%$ between the two values obtained by QAA, although the variance was often as low as $1 \%$. The differences between the two methods were compared, and were found, in all but one case, to be larger than the deviations in the QAA measurements. In addition, in all cases except that one, the $A_{280}$-determined value was larger than the QAA value. 
Table 1

Comparison of the results of protein concentration determinations by the methods of Gill and von Hipple $(\mathrm{GvH})[19]$ and duplicate quantitative amino acid analyses (QAA)

\begin{tabular}{lcrcc}
\hline Protein & $\begin{array}{c}\text { Concentration by } \\
A_{280 \mathrm{~nm}}\end{array}$ & $\begin{array}{c}\text { QAA } \\
\text { average }\end{array}$ & $\begin{array}{c}\text { QAA } \\
\text { SD }\end{array}$ & $\begin{array}{c}\text { Difference } \\
|\mathrm{QAA}-\mathrm{GvH}|\end{array}$ \\
\hline Con A & 10.7 & 9.69 & 0.08 & 1.01 \\
Lysozyme & 9.4 & 8.54 & 0.45 & 0.86 \\
Myoglobin & 7.4 & 7.46 & 0.44 & $\mathbf{0 . 0 6}$ \\
Avidin & 12.3 & 10.01 & 0.04 & 2.29 \\
HSA & 5.5 & 5.22 & 0.24 & 0.28 \\
Ceruloplasmin & 11.4 & 10.16 & 0.10 & 1.12 \\
\hline
\end{tabular}

Note that where the difference between the values obtained by the different methods is less than the standard deviation (SD) of the measurements, the value is in bold italics.

Table 2

Wavelength calibrations showing the peak positions (in $\mathrm{nm}$ ), as measured on different instruments for benzene vapour and a certified holmium oxide filter compared with literature values. The peak position of the positive $\pi \rightarrow \pi^{*}$ exciton peak of the protein myoglobin $(\mathrm{Mb})$ is also included for comparison. Values for $\mathrm{cCD} 1$ are shown before (init) and after (recal) recalibration

\begin{tabular}{|c|c|c|c|c|c|c|c|c|c|c|c|}
\hline & \multirow{2}{*}{$\begin{array}{l}\text { Literature } \\
\text { value }\end{array}$} & \multicolumn{2}{|c|}{ cCD1 } & \multirow[t]{2}{*}{ cCD2 } & \multirow[t]{2}{*}{$\mathrm{cCD} 3$} & \multirow[t]{2}{*}{ cCD4 } & \multirow[t]{2}{*}{ SRCD1 } & \multirow[t]{2}{*}{ SRCD2 } & \multirow[t]{2}{*}{ SRCD3 } & \multirow[t]{2}{*}{ SRCD4 } & \multirow[t]{2}{*}{ SRCD5 } \\
\hline & & init & recal & & & & & & & & \\
\hline Benzene & 241.7 & 240.1 & 241.6 & 241.8 & 241.6 & 241.8 & - & 241.7 & 242.7 & - & - \\
\hline \multirow[t]{2}{*}{ vapour } & 253.0 & 252.0 & 252.8 & 253.1 & 252.9 & 253.1 & - & 253.0 & 253.7 & - & - \\
\hline & 260.1 & 259.1 & 259.8 & 260.1 & 260.0 & 260.0 & - & 260.0 & 260.8 & - & - \\
\hline Holmium & 279.2 & 278.1 & 279.1 & 279.2 & 279.0 & 280.0 & 279.1 & 279.3 & - & 280.6 & 279.1 \\
\hline \multirow[t]{2}{*}{ oxide filter } & 360.9 & 359.4 & 360.3 & 361.6 & 361.0 & 362.0 & 360.7 & - & - & 362.3 & - \\
\hline & 453.7 & 451.7 & 452.5 & 454.0 & 454.0 & 455.0 & - & - & - & & \\
\hline Myoglobin & & 191.6 & 192.3 & 192.2 & 192.0 & 192.6 & 192.0 & 192.2 & 193.0 & 193.1 & 192.0 \\
\hline
\end{tabular}

\subsection{Wavelength comparisons}

The holmium glass filter produces peaks at $279.2 \mathrm{~nm}, 360.9 \mathrm{~nm}$, and $453.65 \mathrm{~nm}$. However most existing SRCD detectors are limited to wavelengths below $350 \mathrm{~nm}$; consequently, only the $279.2 \mathrm{~nm}$ peak could be measured on these instruments. In the UV range, for three of the SRCD instruments the holmium oxide filter gave rise to peaks within $0.1 \mathrm{~nm}$ of the certified wavelength (Table 2). On one conventional instrument this peak was shifted by $+0.8 \mathrm{~nm}$ while the other $\mathrm{cCD}$ instruments were accurate to within $0.2 \mathrm{~nm}$, which is well within instrument specifications. These variances are roughly comparable to the wavelength shifts seen for the $260.1 \mathrm{~nm}, 253.0 \mathrm{~nm}$ and $241.7 \mathrm{~nm}$ benzene peaks. One SRCD instrument for which no holmium oxide measurement could be obtained, exhibited shifts ranging between +1.0 and $+0.7 \mathrm{~nm}$ for the benzene peaks. The results are shown in Fig. 2 as a plot of expected versus measured wavelength. In the UV range the biggest difference (after re-calibration) was $1.0 \mathrm{~nm}$. However, the wavelength shifts increased in the visible range to deviations from the literature value of as much as $1.3 \mathrm{~nm}$ on one instrument and a net difference between instruments of $2.5 \mathrm{~nm}$. Very similar trends were seen for the benzene and holmium standards. Benzene has an advantage of having several measurable peaks in the UV region where most protein peaks are, but it has the disadvantage of requiring the ability to set a small bandwidth for the measurement of its sharp peaks. 


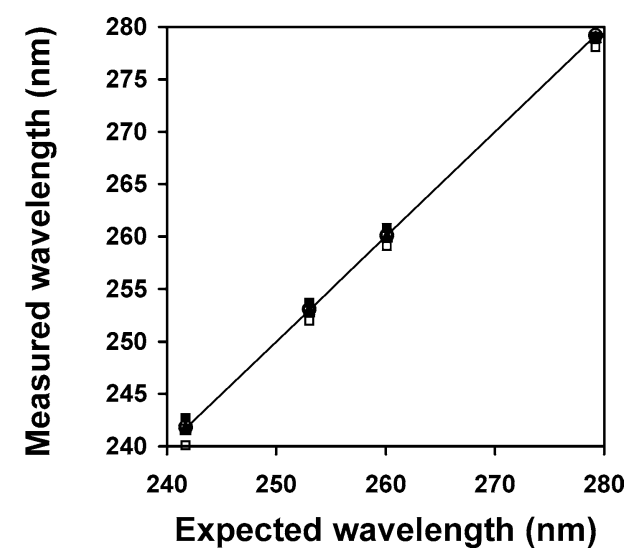

Fig. 2. Plot of expected (literature values) versus measured wavelength positions (in $\mathrm{nm}$ ) showing variations between different instruments in the absorption peaks of a certified holmium oxide glass filter and benzene vapour. The open symbols correspond to conventional CD instruments, the filled symbols to SRCD instruments.

Table 3

Effect of (artificial) wavelength shifts on calculated secondary structure contents for several proteins

\begin{tabular}{lccc}
\hline Protein & $\begin{array}{c}\text { Wavelength } \\
\text { shift }(\mathrm{nm}) \\
\text { applied }\end{array}$ & $\begin{array}{c}\text { Calculated } \\
\text { helix content } \\
(\%)\end{array}$ & $\begin{array}{c}\text { Calculated } \\
\text { sheet content } \\
(\%)\end{array}$ \\
\hline Myoglobin & 0 & 75 & 0 \\
& +1 & 77 & 0 \\
HSA & -1 & 69 & 1 \\
& 0 & 72 & 1 \\
Lysozyme & +1 & 72 & 2 \\
& -1 & 70 & 1 \\
& 0 & 38 & 14 \\
Con A & +1 & 39 & 15 \\
& -1 & 36 & 13 \\
& 0 & 9 & 39 \\
Avidin & +1 & 7 & 42 \\
& -1 & 11 & 37 \\
& 0 & 1 & 53 \\
Ceruloplasmin & +1 & 1 & 52 \\
& -1 & 1 & 51 \\
& 0 & 3 & 33 \\
& +1 & 4 & 34 \\
\hline \multirow{4}{*}{ (1) } & -1 & 4 & 32 \\
\hline
\end{tabular}

The wavelength variations seen in the standards are also reflected in the spectra measured for myoglobin on these instruments where the difference in the measured position of the positive $\pi \rightarrow \pi^{*}$ exciton peak at $\sim 192 \mathrm{~nm}$ is as much as $1.1 \mathrm{~nm}$ on different instruments. To illustrate the effect of this type of wavelength shift on secondary structure analyses (without other factors such as optical rotation magnitude contributing to the difference) the CD spectra of a number of proteins were artificially shifted by $+1 \mathrm{~nm}$ and $-1 \mathrm{~nm}$ and the calculated secondary structures from the resulting spectra were compared. 
Table 3 shows that wavelength shifts can have a small but significant effect on the calculated helix and sheet content of proteins. For example a shift of $+1 \mathrm{~nm}$ leads to a small increase in the predicted helix content in the mostly helical protein myoglobin (from $75 \%$ to $77 \%$ ), while a shift of $-1 \mathrm{~nm}$ causes a decrease to 69\%. It appears generally (but not always) that for the mostly helical proteins (myoglobin, HSA and lysozyme) shifts of $+1 \mathrm{~nm}$ tend to increase calculated helical content and decrease sheet content; the converse results are true for shifts of -1 . The effects on the mostly sheet proteins are less pronounced, but often +1 shifts lead to increased sheet contents and -1 shifts lead to decreased sheet contents. The effects of errors in peak positions are not nearly as dramatic as those produced by errors in spectral magnitude [8], nevertheless, they have discernable consequences on the calculated structures.

\section{Conclusion}

A major potential source of magnitude error, incorrect cell pathlength, is more commonly found for short pathlength cells. If a $1 \mathrm{~mm}$ cell deviates by $10 \mu \mathrm{m}$, this only gives rise to an error of $1 \%$; however if a $10 \mu \mathrm{m}$ cell deviates by $4 \mu \mathrm{m}$, as was found in this study, this results in a $40 \%$ error, which is unacceptable for quantitative experiments. For pathlengths between $50 \mu \mathrm{m}$ and $1 \mathrm{~mm}$, carefully prepared solutions of potassium chromate can be used for calibration. But for very short pathlength cells, the absorbance values are too small for accurate determinations. For these cells the interference fringe method gives a more precise measurement, with an error of $\leqslant 0.2 \mu \mathrm{m}$. This method cannot, however, be used for cells with pathlengths longer than $\sim 20 \mu \mathrm{m}$ for practical reasons, based on the number of resolvable fringes.

Similarly, it is essential to have accurate values for protein concentrations. The best, although most costly, results are obtained by quantitative amino acid analysis. This method requires only small amounts of sample ( $\sim 1$ nmole) and involves the hydrolysis of the protein into its constituent residues, which are then derivitised for detection by fluorescence and separated by column chromatography. If the sequence of the protein is known, then the quantitation of well-recovered residues, mainly those with small hydrophobic side chains, in the presence of a standard, provides an accurate measure at the concentration of the protein. If the sequence is not accurately known, there are alternative methods of recovering all residues including the most labile ones such as tryptophan [16]. In a comparison of absorbance methods versus QAA for bovine serum albumin, it was found that other methods including the use of $A_{280 \mathrm{~nm}}$ tended to overestimate the concentration of the protein, probably due to undetected bound salts and water molecules [17], a trend which was also seen for the other proteins examined in the present study (Table 2). Absorption methods can also be affected by $\mathrm{pH}$, temperature, buffer composition and light scattering, not accounted for in the standard empirical calculation methods. Obviously gravimetric methods, which are often relied on instead, can also be severely affected by other components present such as salts, buffers, etc., which contribute to the apparent amount weighed out, and are subject to even larger errors.

The effect of monochromator misalignment may appear small when visually comparing spectra but wavelength shifts of as little as $1 \mathrm{~nm}$ can have a significant effect on secondary structure analyses. It should be noted that an apparent shift in wavelength can also result from setting the spectra acquisition time constant too low or having a protein concentration that is too high [8]. It is necessary to check the calibration of an instrument at several wavelengths including at least one in the far UV where the protein amide absorption occurs. Benzene vapour, which is usually used to determine the wavelength resolution of an instrument, provides a cheap method to calibrate wavelength. Although the useful range is small, 
between 240 and $270 \mathrm{~nm}$ the peaks are much sharper than those of the holmium oxide glass filter, which are at higher wavelengths.

Acetone vapour has been suggested as an alternative wavelength standard for the far UV, as it gives rise to peaks between $195 \mathrm{~nm}$ and $166 \mathrm{~nm}$ [18]. Measurements of vapour from $5 \mu 1$ of acetone in a $1 \mathrm{~mm}$ cell produced resolvable peaks in the HT signal at $\sim 190.7 \mathrm{~nm}, \sim 195 \mathrm{~nm}$ when measured on a conventional machine and one SRCD instrument (data not shown). However, on conventional instruments the slits need to be fully open at lower wavelengths to increase the light flux to enable the measurement and this creates uncertainty in the wavelengths measured. For SRCD instruments, calibration at much lower wavelengths can be achieved by observing a series of electric dipole forbidden nitrogen transitions between $145 \mathrm{~nm}$ and $127 \mathrm{~nm}$ [19]. In this study, six distinct peaks were observed in the HT signal at 145, 142, 136, 133, 130 and $127 \mathrm{~nm}$ using two SRCD instruments (data not shown).

In summary, in this paper a number of parameters important for producing accurate and reproducible CD spectra were examined. Methods for improving the accuracy and comparability of CD spectral magnitudes and peak positions were presented. Hence, this paper provides practical guidance for "good practice" in the measurement of CD data.

\section{Acknowledgements}

This work was supported by grants B13586 and B14225 from the BBSRC to B.A.W. and grant B02959 to R.W. Janes. Beamtime at the SRS was supported by grants from the CLRC to B.A.W. Beamtime access at ISA was enabled by the European Community - Research Infrastructure Action under the FP6 "Structuring the European Research Area" Programme to Soren Pape Moller (Aarhus University). Some research was carried out at the National Synchrotron Light Source, Brookhaven National Laboratory, which is supported by the U.S. Department of Energy, Division of Materials Sciences and Division of Chemical Sciences, under Contract No. DE-AC02-98CH10886. J.G.L. was supported by a BBSRC CASE studentship with GlaxoSmithKline, and A.J.M. was supported by an MRC studentship. We thank the following for technical assistance at the SRCD beamlines: Dr. Soren Vronning Hoffmann (UV1 beamline, ISA), Dr. David Clarke (CD12 beamline, SRS Daresbury), John Trunk (U9b and U11 beamlines, NSLS), and Dr. Peter Baumgaertel (beamline 3m_NIM1_C, BESSY2). We also thank Dr. Robert W. Janes (Queen Mary, Univ. of London) and Dr. Alison Rodger (Warwick University) for helpful discussions and access to their CD instruments and Dr. Janes for help with some of the SRCD measurements. We thank Peter Sharratt of the PNAC facility, University of Cambridge, for the quantitative amino acid analyses.

\section{References}

[1] J.C. Sutherland, E.J. Desmond and P.Z. Takacs, Versatile spectrometer for experiments using synchrotron radiation at wavelengths greater than $100 \mathrm{~nm}$, Nucl. Instr. Meth. 172 (1980), 195-199.

[2] P.A. Snyder and E.M. Rowe, The first use of synchrotron radiation for vacuum ultraviolet circular dichroism measurements, Nucl. Instr. Meth. 172 (1980), 345-349.

[3] B.A. Wallace, Synchrotron radiation circular dichroism spectroscopy as a tool for investigating protein structures, J. Synch. Rad. 7 (2000), 289-295.

[4] B.A. Wallace and R.W. Janes, Synchrotron radiation circular dichroism spectroscopy of proteins: Secondary structure, fold recognition and structural genomics, Curr. Opin. Chem. Biol. 5 (2001), 567-571.

[5] B.A. Wallace, F. Wien, A.J. Miles, J.G. Lees, P. Evans, S.V. Hoffman, G.J. Wistow and C. Slingsby, Biomedical applications of synchrotron radiation circular dichroism spectroscopy: Identification of mutant proteins associated with disease and development of a reference database for fold motifs, Faraday Discussions 126 (2004), 237-243. 
[6] A.J. Miles, F. Wien, J.G. Lees, A. Rodger, R.W. Janes and B.A. Wallace, Calibration and standardisation of synchrotron radiation circular dichroism and conventional circular dichroism spectrophotometers, Spectroscopy 17 (2003), 653-661.

[7] J.G. Lees, B.R. Smith, F. Wien, A.J. Miles and B.A.Wallace, CDtool-An integrated software package for circular dichroism spectroscopic data processing, analysis and archiving, Anal. Biochemistry 332 (2004), 285-289.

[8] A.J. Miles, L. Whitmore and B.A. Wallace, Spectral magnitude effects on the analyses of secondary structure from circular dichroism spectroscopic data, Protein Sci., in press.

[9] S.C. Gill and P.H. von Hipple, Calculation of protein extinction coefficients from amino acid data, Anal. Biochemisty 182 (1989), 319-326.

[10] J.P. Hennessey, Jr. and W.C. Johnson, Jr., Experimental errors and their effects on analyzing circular dichroism spectra of proteins, Anal. Biochemistry 125 (1982), 177-188.

[11] F.M. Garforth and C.K. Ingold, Excited states of benzene. Part II. Analysis of the first ultraviolet band system of the absorption spectrum of benzene, J. Chem. Soc. (1948), 417.

[12] L. Whitmore and B.A. Wallace, DICHROWEB, an online server for protein secondary structure analyses from circular dichroism spectroscopic data, Nucleic Acids Research 32 (2004), W668-W673.

[13] I.H.M. van Stokkum, H.J.W. Spoelder, M. Bloemendal, R. Van Grondelle and F.C.A. Groen, Estimation of protein secondary structure and error analysis from CD spectra, Anal. Biochemistry 191 (1990), 110-118.

[14] N. Sreerama and R.W. Woody, Estimation of protein secondary structure from circular dichroism spectra: Comparison of CONTIN, SELCON and CDSSTR methods with an expanded reference set, Anal. Biochemistry 282 (2000), 252-260.

[15] G.W. Haupt, An alkaline solution of potassium chromate as a transmittancy standard in the ultraviolet, J. Res. Natl. Bureau of Standards 48 (1952), 414-423.

[16] J.C. Anders, Advances in amino acid analysis, Biopharm. 15 (2002), 32-39.

[17] J.C. Anders, B.F. Parten, G.E. Petrie, R.L. Marlow and J.E. McEntire, Using amino acid analysis to determine absorptivity constants, Biopharm. 16 (2003), 30-37.

[18] J. Lake and A.J. Harrison, Absorption of acyclic oxygen compounds in the vacuum ultraviolet. III. Acetone and acetaldehyde, J. Chem. Phys. 30 (1959), 361-362.

[19] A. Lofthus and P.H. Krupenie, The spectrum of molecular nitrogen, J. Phys. Chem. 6 (1977), 113-307. 


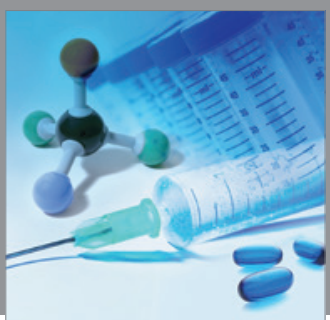

International Journal of

Medicinal Chemistry

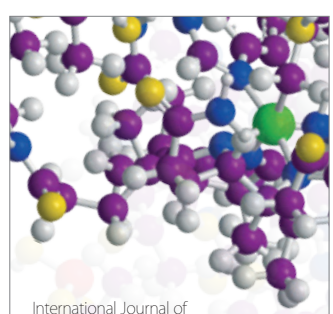

Carbohydrate Chemistry

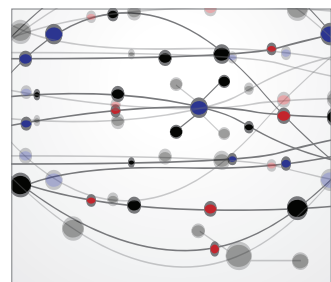

The Scientific World Journal
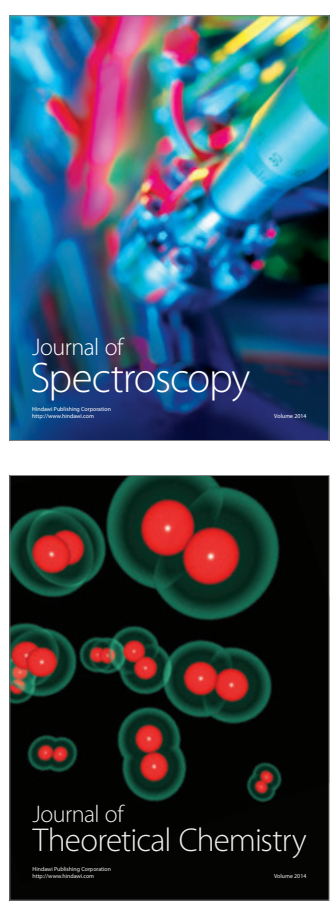
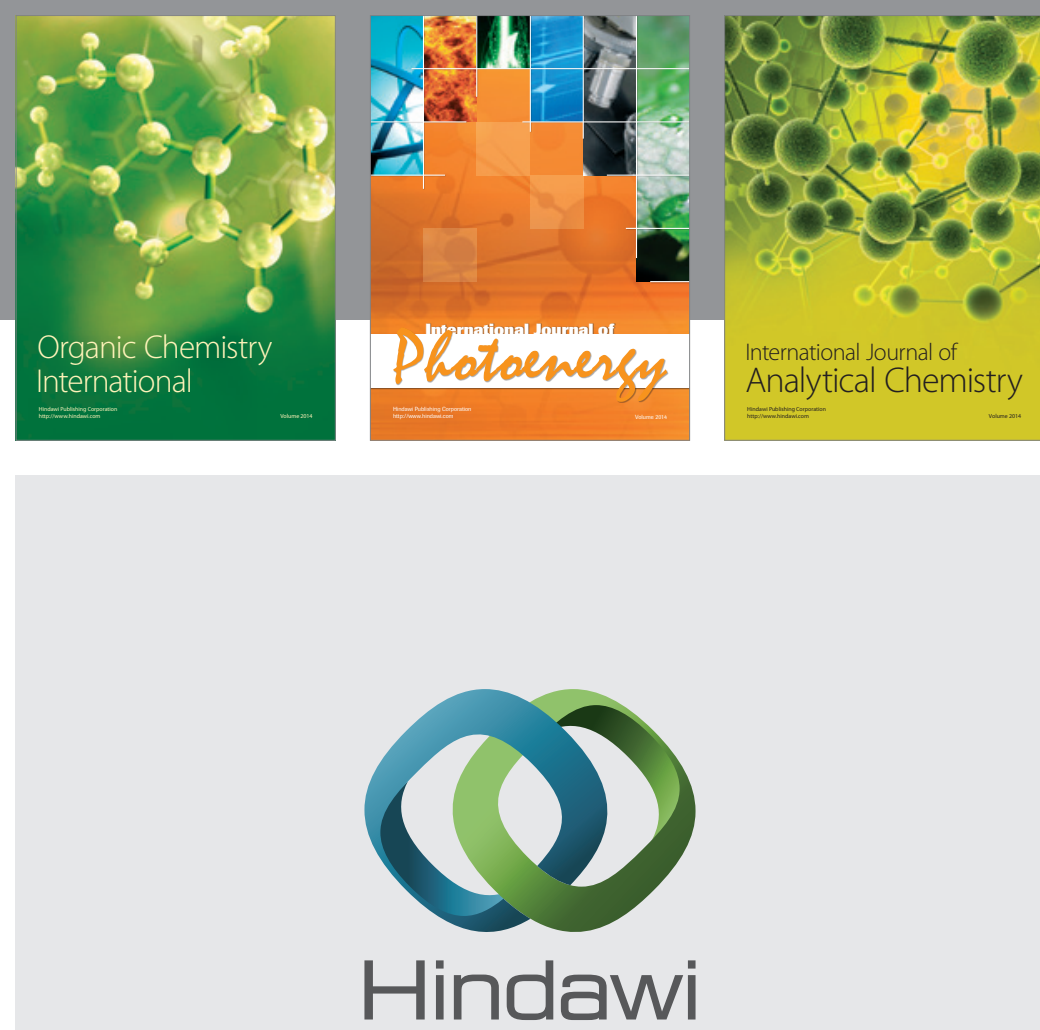

Submit your manuscripts at

http://www.hindawi.com
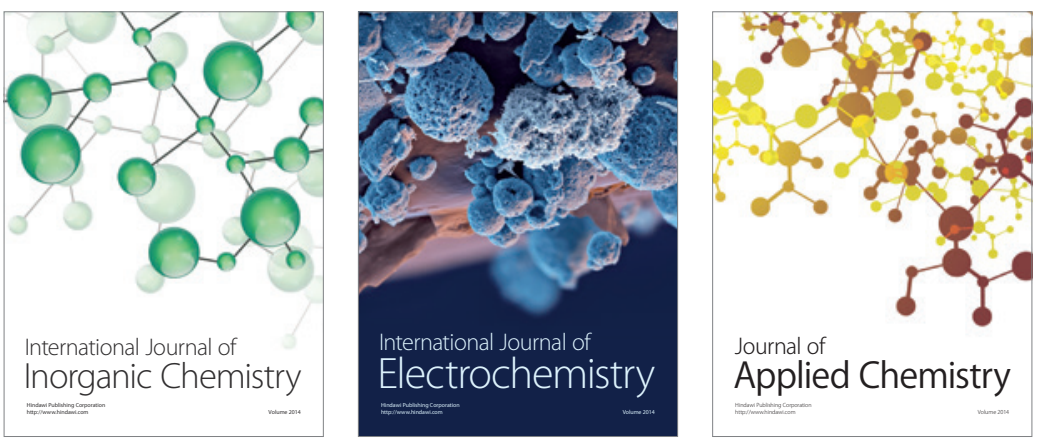

Journal of

Applied Chemistry
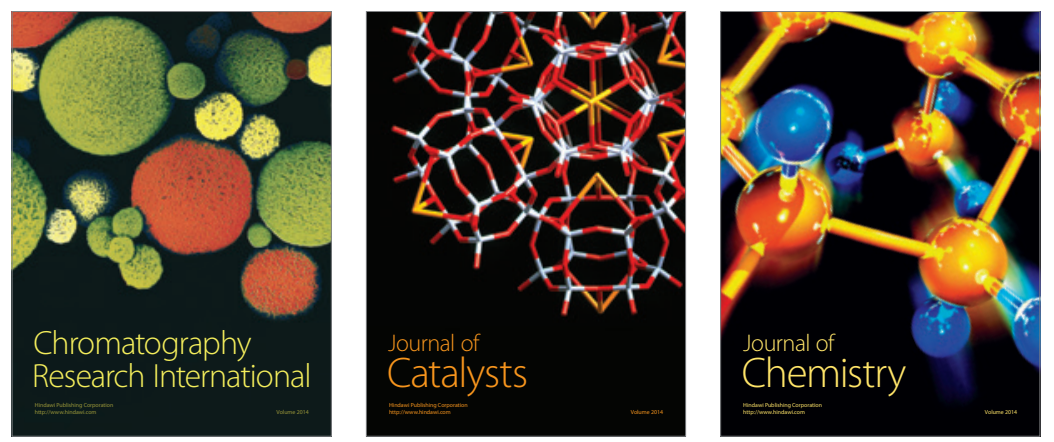
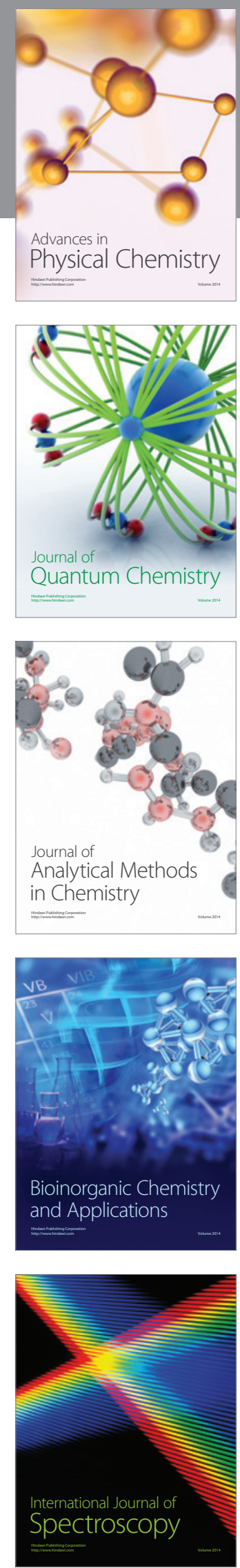